\title{
Determinación de anticuerpos frente al antígeno A-60 para el diagnóstico de la infección por M. tuberculosis. Una herramienta útil para la racionalización de la quimioprofilaxis en pacientes VIH
}

\author{
J.R. BLANCO, V. MARTÍNEZ DE ARTOLA, V. IBARRA, L. ROSEL, R. GÓMEZ- \\ CADIÑANOS, J.A. OTEO
}

Servicio de Medicina Interna y Enfermedades Infecciosas. Hospital de La Rioja. Logroño

\section{RESUMEN}

Objetivo: Identificar la infección por M. tuberculosis en pacientes VIH mediante la determinación del PPD y de un test de ELISA para la detección de anticuerpos frente al antigeno A-60 de M. bovis.

Métodos: Se han estudiado 116 pacientes VIH, clasificados de acuerdo a los CDC. El estado de infección por M. tuberculosis se diagnosticó mediante el PPD (RT-23) y el estado de anergia fue estimado mediante Multitest CMI (Multitext ${ }^{\oplus}$ ). La presencia de anticuerpos circulantes del tipo IgG contra el antigeno A-60 fueron analizados por un método de ELISA. El curso clínico de los pacientes fue analizados durante 24 meses.

Resultados: Se observó una serología positiva (A-60) en el 52,58\% de los pacientes, coincidiendo con el 70,45\% de los pacientes PPD (+) $(\mathrm{p}<0,003)$. Esta observación estadística fue encontrada al analizar todos los pacientes, y cuando se analizaban aquellos con >500 linfocitos CD4 El Multitext ${ }^{\oplus}$ fue positivo en el $11,11 \%$ de los pacientes PPD (-), todos ellos sin anticuerpos frente al antígeno A-60. Sin embargo, el 42,2\% de los pacientes PPD y Multitext ${ }^{\circledast}(-)$ presentaban anticuerpos frente al antígeno A-60. El seguimiento de los pacientes durante 24 meses permitió la detección de 7 nuevos casos de tuberculosis pulmonar, 4 de ellos presentaban una serología positiva para el antígeno A-60, y 4 fueron anérgicos.

Conclusiones: Los pacientes VIH con infección por M. tuberculosis presentan anticuerpos circulantes frente al antígeno A-60 de $M$. bovis con independencia de su estado de inmunidad. En los pacientes anérgicos, la presencia de estos anticuerpos puede ser una herramienta útil para racionalizar la profilaxis frente a M. tuberculosis. La seropositividad (A-60) indica, al igual que el PPD un contacto previo con M. tuberculosis.

PALABRAS CLAVE: Antígeno A-60. VIH. Tuberculosis. Infección $M$. tuberculosis. Anergia.

\begin{abstract}
DETERMINATION OF ANTIBODIES AGAINST A-60 ANTIGEN FOR DIAGNOSIS OF M. TUBERCULOSIS INFECTION. AN USEFUL TOOL FOR RATIONALIZING OF CHEMOPROPHYLAXIS IN HIV PATIENTS
\end{abstract}

\section{ABSTRACT \\ Objective: To identify M. tuberculosis infection in HIV patients by} use of PPD and an ELISA test detecting the presence of antibodies against antigen $A-60$ of $\mathrm{M}$. bovis.

Methods: 116 incoming HIV patients were classified according to the $C D C$. The status of $\mathrm{M}$. tuberculosis infection was probed with PPD (RT-23) and the state of anergy was estimated by a Multitest CMI (Multi text $\left({ }^{B}\right)$. The presence of circulating antibodies of the $\operatorname{Ig} G$ class against antigen A-60 was analysed by an ELISA. The clinical course of the patients was followed for 24 month.

Results: A positive serology (A-60) was found in $52.58 \%$ of the patients, coinciding with $70.45 \%$ of the positive PPD ( $p<0.003)$. This statical observation was found when we studied all of the individuals, and those with more than 500 CD4 lymphocytes. The Multitext ${ }^{\circledR}$ was positive in $11.11 \%$ of the patients with PPD (-), all of them without anti bodies against A-60. However, $42.2 \%$ of the aptients who were PPD and Multitext ${ }^{\circledR}(-)$, had circulating antibodies against A-60. The following up of the patients during 24 months allowed the detection of 7 news cases of pulmonary tuberculosis, 4 of them had a positive A-60 serology, and 4 were anergy.

Conclusions: HIV patients with M. tuberculosis infection have circu lating antibodies against A-60 antigen of M. bovis with independence of their immunity status. In anergic patients the presence of this antibodies can be an useful tool for rationalizing the prophylaxis against M. tuberculosis. A seropositivity (A-60) indicates as well as does PPD a previous contact with $\mathrm{M}$. tuberculosis.

KEY WORDS: A-60 antigen. HIV. Tuberculosis. M. tuberculosis infec tion. Anergy.

Blanco JR, Martínez de Artola V, Ibarra V, Rosel L, Gómez-Cadiñanos R, Oteo JA. Determinación de anticuerpos frente al antígeno A-60 para el diagnóstico de la infección por M. tuberculosis. Una herramienta útil para la racionalización de la quimioprofilaxis en pacientes VIH. An Med Interna (Madrid) 2001; 18: 127-131.

Trabajo aceptado: 20 de Marzo de 2000

Correspondencia: José Antonio Oteo. Servicio de Medicina Interna y Enfermedades Infecciosas. Hospital de La Rioja. Avda. de Viana, 1 - 26001 Logroño (La Rioja). 


\section{INTRODUCCIÓN}

La infección por el virus de la inmunodeficiencia humana (VIH), se considera en la actualidad el principal factor de riesgo para el desarrollo de tuberculosis (TB) $(1,2)$. El problema alcanza tal magnitud que el elevado número de personas coinfectadas por el virus VIH y Mycobacterium tuberculosis en el mundo ha determinado que se recomiende excluir la infección por el VIH en pacientes con TB activa, y valorar el estado de infección tuberculosa (IT) en pacientes infectados por el VIH (1-4).

Dentro de las múltiples estrategias que tenemos para romper la cadena epidemiológica de la IT y de la enfermedad tuberculosa, la quimioprofilaxis antituberculosa (QP) se ha mostrado eficaz para evitar el desarrollo de enfermedad tuberculosa (5).

A la hora de estudiar la IT la prueba de la tuberculina (PPD) mediante la intradermorreacción de Mantoux es el método más sensible y más barato para su diagnóstico. El problema es que su sensibilidad disminuye en los pacientes infectados por VIH debido a su inmunosupresión celular (6-8). Por este motivo, y porque algunos investigadores han observado un riesgo similar para el desarrollo de TB en los pacientes PPD (+) que en los PPD (-) con anergia cutánea, la recomendación de QP se hace extensiva a todos los grupos con una elevada prevalencia de IT (4,9-12). Dado que la patogénesis de la enfermedad tuberculosa indica que un alto porcentaje de pacientes desarrolla TB por reactivación de su IT, se hace necesario conocer con exactitud aquellos pacientes con IT.

El propósito de este trabajo es identificar, dentro de los pacientes infectados por el VIH, la IT mediante la realización del PPD y de un test de ELISA que es capaz de detectar la presencia de anticuerpos frente al antígeno A-60 de M. bovis (A-60).

\section{PACIENTES Y MÉTODO}

\section{PACIENTES}

Entre el 1 de noviembre de 1995 y el 31 de octubre de 1996, hemos realizado un estudio prospectivo en los pacientes infectados por el VIH atendidos en el Servicio de Medicina Interna y Enfermedades Infecciosas del Hospital de La Rioja que acuden regularmente a las Consultas. El área sanitaria de influencia comprende una población de unos 65.000 habitantes, además del Centro Penitenciario y de aquellos pacientes infectados por el VIH que desean ser atendidos en nuestro Servicio.

Se recogieron los siguientes datos de los pacientes: edad, sexo, vía de adquisición de la infección por el VIH, clasificación de los pacientes VIH según los CDC (13), QP previa con isoniazida, historia previa de TB, desarrollo de TB durante el período de seguimiento ( 24 meses) y cuantificación del número total de linfocitos y de linfocitos $\mathrm{CD}_{4}$. Los pacientes con TB activa fueron excluidos del estudio.

\section{MÉTODO}

Pruebas cutáneas: A todos los pacientes objeto del estudio se les realizó estudio de tuberculina-PPD mediante la téc- nica de Mantoux (2 unidades RT/PPD-23) y lectura a las 72 horas mediante la técnica de Sokal, según recomendación del Consenso Español (5). En aquellos que resultaban negativos (diámetro transversal de induración $<5 \mathrm{~mm}$ ), se procedió a la administración de un Multitext ${ }^{\circledR}$ (Multitest CMI, Rohne-Poulenc Farma, SAE, Madrid) que determina la presencia de inmunidad celular frente a diferentes antígenos (toxoide tetánico, toxoide diftérico, antígeno Streptococcus, tuberculina vieja, glicerina [control], antígeno Candida albicans, antígeno Trichophyton y antígeno Proteus). Para su administración se siguieron las recomendaciones de la casa comercial. Se consideró como positiva la presencia de induración $>$ o igual a 2 mm en cualquiera de los puntos de inoculación intradérmica en lectura efectuada a las 48 horas. Para evitar errores de interpretación y lectura, la administración de las pruebas intradérmicas y su posterior lectura fue realizada siempre por dos enfermeras de nuestro Servicio bien entrenadas y con amplia experiencia en este campo. No se consideró la administración de otro PPD para evaluación de efecto Booster dada la escasa prevalencia en este colectivo del citado efecto $(8,14)$.

Investigación de anticuerpos frente a $M$. tuberculosis: En el mismo día de realización de la prueba del PPD se procedió a la extracción mediante venopunción $10 \mathrm{cc}$ de sangre que fue centrifugada para la separación de suero y posterior congelación a menos $70^{\circ} \mathrm{C}$. Posteriormente y una vez obtenidos todos los sueros objetos del estudio se descongelaron y se investigó la presencia de anticuerpos frente a $M$. tuberculosis mediante una técnica de ELISA que investiga la presencia de anticuerpos frente el antígeno A-60 de M. bovis (Anda Biologicals, Estrasburgo, Francia, distribuido en España por Biosell) siguiendo las recomendaciones de la casa comercial. Como punto de discriminación entre positivo y negativo se tomaron las 100 U.I. que es el nivel de anticuerpos que recomienda la casa comercial para niños e inmunodeprimidos.

Estadística: La comparación de proporciones se realizó con la prueba del chi-cuadrado, excepto cuando alguno de los efectivos esperados fue menor a cinco. En este caso, se empleó la prueba exacta de Fisher. La comparación de medias se realizó mediante la prueba t de Student, previa comprobación del supuesto de homogeneidad de varianzas. Todas las pruebas fueron bilaterales, considerando que el resultado era significativo cuando el riesgo alfa fue inferior a $5 \%$.

Los cálculos se efectuaron con ayuda del programa informático SPSS para Windows, versión 6.1.2.

\section{RESULTADOS}

En total se han estudiado 116 pacientes, 86 varones $(74,1 \%)$ y 30 mujeres $(25,9 \%)$. La media de edad fue de 32,54 años (intervalo de 20 a 71). La principal práctica de riesgo para la infección por el VIH fue el uso de drogas por vía parenteral (UDVP) $(81,9 \%)$, seguido de las prácticas sexuales (heterosexuales $14,7 \%$, homosexuales $2,6 \%$ y bisexuales $0,9 \%)$.

La distribución de los pacientes VIH según las diferentes categorías establecidas por los CDC fue la siguiente: categoría 1 (> o igual a 500 linfocitos $C D_{4}$ ) $25,9 \%$; categoria $234,5 \%$, y categoría 3 ( $<200$ linfocitos $\mathrm{CD}_{4}$ ) 39,6\%. La media de linfocitos $\mathrm{CD}_{4}$ fue de 339,09 (intervalo de 4 a 999). Se encontraban en estadio de SIDA (clasificación de los CDC) un 42,2\% de 
los pacientes. De las diferentes afecciones definitorias de SIDA, llama la atención el alto porcentaje de TB (40,8\%), seguido de la candidiasis esofágica $(24,5 \%)$, y de la neumonía por $P$. carinii $(22,4 \%)$.

Del total de la muestra, $44(37,9 \%)$ presentaban un PPD (+) (Tabla I), disminuyendo su porcentaje de positividades a medida que lo hacía la inmunidad celular. De acuerdo a la clasificación de los CDC, el 53\% estaban en la categoría 1, el $50 \%$ en la categoría 2 y el 17,4\% en la categoría 3, siendo la asociación lineal altamente positiva $(\mathrm{p}<0,001)$ (Tabla II) (Figura 1). Sólo 8 pacientes con un PPD (-) $(11,11 \%)$ presentaban un Multitext ${ }^{\circledast}$ positivo, y de éstos, ninguno presentaba anticuerpos frente al A-60. Al comparar la cifra de linfocitos $\mathrm{CD}_{4}$ en pacientes con un PPD o Multitext ${ }^{\oplus}$ positivo, frente a aquellos con ambas pruebas negativas, vemos como los primeros presentan una media de 466,9 (DE 251,8) linfocitos $\mathrm{CD}_{4}$, frente a los 233,6 (DE 218,3) $\mathrm{CD}_{4}$ de los últimos, con una diferencia significativa $(\mathrm{p}<0,001)$. De los pacientes con PPD y Multitext ${ }^{\oplus}$ negativos, el $42,2 \%$ presentaba un A-60 positivo.

\section{TABLA I}

RELACIÓ N ENTRE EL TEST DE LA TUBERCULINA (PPD) Y LA PRESENCIA DE ANTICUERPOS FRENTE AL ANTÍGENO A-60

\begin{tabular}{lcc} 
& PPD (+) & PPD (-) \\
\hline A-60 (+) & $31(26,7 \%)$ & $30(25,9 \%)$ \\
A-60 (-) & $13(11,2 \%)$ & $42(36,2 \%)$ \\
\hline
\end{tabular}

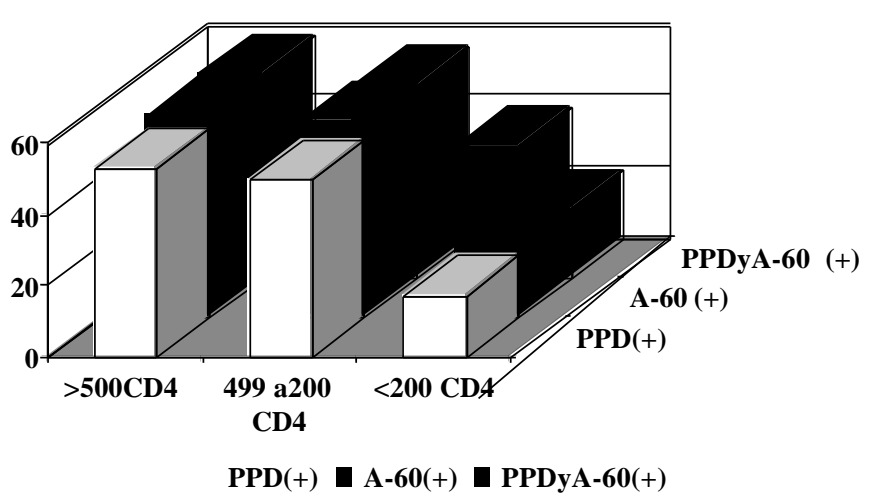

Fig. 1. Porcentaje de pacientes con test de tuberculina (PPD) y anticuerpos frente al antígeno A-60, según el número de linfocitos CD4.

En el total de nuestros pacientes observamos que el $52,58 \%$ presentaban una serología (anticuerpos frente al A$60)$ positiva, coincidiendo con un PPD (+) en el 70,45\%, con una asociación lineal altamente significativa $\mathrm{p}<0,003$. La probabilidad medida mediante la Odds Ratio (OR) de que un paciente con un PPD $(+)$ presente anticuerpos frente al A-60 es de un 3,34. Esta asociación estadística se mantiene cuando estudiamos los pacientes infectados por el VIH del estadio 1
TABLA II

RELACIÓ N ENTRE EL TEST DE LA TUBERCULINA (PPD) Y LA PRESEN CIA DE ANTICUERPO S FRENTE AL ANTÍGENO A-60, SEGÚN EL NÚMERO DE LINFOCITOS $\mathrm{CD}_{4}$

\begin{tabular}{crcr}
\hline & $>500 \mathrm{CD}_{4}$ & $499{\mathrm{a} 200 \mathrm{CD}_{4}}$ & $<200 \mathrm{CD}_{4}$ \\
\hline PPD (+) & $16(53,3 \%)$ & $20(50 \%)$ & $8(17,4 \%)$ \\
$(-)$ & $14(46,6 \%)$ & $20(50 \%)$ & $38(82,6 \%)$ \\
& & & \\
A-60 (+) & $17(56,7 \%)$ & $22(55 \%)$ & $22(47,8 \%)$ \\
$(-)$ & $13(43,3 \%)$ & $18(45 \%)$ & $24(52,2 \%)$ \\
PPD y A-60 (+) & $12(57,1 \%)$ & $14(53,8 \%)$ & $5(19,2 \%)$ \\
$(-)$ & $9(42,8 \%)$ & $12(46,2 \%)$ & $21(80,8 \%)$ \\
\hline
\end{tabular}

(OR 5,4; Intervalo de Confianza del 95\% [IC95\%] de 1,2 a $26,05)$, no así en los pacientes con mayor deterioro inmunológico en los que no encontramos asociación estadística; grupo 2 (OR 3,5; IC95\% de 0,95 a 12,97) y grupo 3 (OR 2,06; IC95\% de 0,43 a 9,87$)$.

Del total de los pacientes con anticuerpos frente al antígeno A-60, un $15,38 \%$ presentaba cifras $>300$ U.I., y de ellos el $50 \%$ habían presentado con anterioridad tuberculosis o la han desarrollado durante el seguimiento.

Durante los 24 meses de seguimiento, 7 pacientes han desarrollado TB pulmonar $(6,03 \%)$. De los 7 pacientes, 4 presentaban una serología positiva frente al A-60, y otros 4 eran anérgicos.

\section{DISCUSIÓN}

Como se ha comentado en la introducción, no existe una prueba sensible y específica para la investigación de la IT en el paciente inmunodeprimido por el virus VIH. Así, en diferentes trabajos se demuestra que la sensibilidad del PPD disminuye a medida que lo hace el número de linfocitos $\mathrm{CD}_{4}$; así, según diferentes autores, el grado de anergia oscila entre el $25 \%$ y el $75 \%(6-8,15,16)$. En nuestro estudio, y como era de esperar, también hemos observado un aumento del número de pacientes con un PPD (-) a medida de que disminuye el número de linfocitos $\mathrm{CD}_{4}(\mathrm{p}<0,001)$. Así, en los pacientes con $<200$ linfocitos $\mathrm{CD}_{4}$ el porcentaje de pacientes con IT puesto de manifiesto mediante el PPD fue del 17,4\% frente al 53,3\% de los pacientes con $>500$ linfocitos $\mathrm{CD}_{4}$.

Aunque el objetivo principal del presente trabajo no era evaluar la utilidad del PPD en la detección de la IT, no queremos pasar por alto el elevado porcentaje de pacientes con PPD (+) $(39,8 \%)$ que presenta nuestra cohorte, muy superior al encontrado por otros autores en España $(8,9)$. Posiblemente este alto porcentaje se debe a que la mayor parte de nuestros pacientes $(81,9 \%)$ son o han sido UDVP, colectivo en el que se ha encontrado una mayor prevalencia de IT $(7,9,16)$. Además nuestro Hospital atiende la zona menos favorecida desde el punto de vista social y económico de la Rioja, así como la cárcel.

Desde hace años, y dada la dificultad del diagnostico de la enfermedad tuberculosa en determinadas circunstancias y 
colectivos, se están buscando pruebas de diagnóstico rápido para la detección de enfermedad tuberculosa (17). Entre ellas la determinación de anticuerpos frente al A-60 extraído del citoplasma de $M$. bovis ha suscitado la realización de numerosos trabajos, que podrían resumirse en que la técnica resulta específica, pero escasamente sensible (18-23). Existen en la literatura muy pocos trabajos que se hayan marcado como objetivo relacionar la IT con la presencia de anticuerpos frente al A-60. Algunos autores no encuentran relación entre la misma y la positividad de la IgG o IgM frente al A-60, rechazando su uso en el diagnóstico de la IT $(20,24,25)$. Por contra, Saz y Ladrón de Guevara (26) han demostrado una correlación entre los PPD (+) y la respuesta a IgG frente al A-60, deduciendo una relación directa entre ambas.

Uno de los problemas que suscita la técnica es la determinación del umbral o punto de corte que se debe tomar para considerar que la presencia de anticuerpos frente al A-60 es indicativa de enfermedad y/o de infección. En nuestro trabajo, dado que la población estudiada está inmunodeprimida y de que tratábamos de identificar la IT y no la enfermedad, decidimos tomar como valor de referencia las 100 U.I. Para ello nos hemos apoyado en las recomendaciones de la casa comercial y en los trabajos de Querol y colaboradores (24), quienes para el diagnóstico de enfermedad tuberculosa con 100 U.I. lograban una sensibilidad del 57\%, inferior a la encontrada por nosotros $(70,5 \%)$, y una especificidad del $91 \%$ claramente superior a la encontrada en nuestro trabajo (58,3\%). Así, en nuestra experiencia la determinación de anticuerpos frente al A-60 tiene escaso valor como técnica de diagnóstico de enfermedad tuberculosa, no así para el diagnóstico de IT. No obstante uno de los problemas del antígeno A-60 es que se encuentra en todas las micobacterias, y presenta reacciones cruzadas con otros microorganismos, incluyendo Nocardia spp., Corynebacterium spp. y Leishaminias $(24,27)$, que se han sido excluídas razonablemente en nuestros pacientes.

Como se ha expuesto en los resultados, el 52,58\% de todos nuestros pacientes presentaban una serología positiva frente al A-60, lo que coincidía con un PPD (+) en el 70,45\% (p<0,003 y OR 3,34). Este resultado, que confirma los trabajos de Saz y Ladrón de Guevara (26) ofrece un paralelismo aceptable entre las dos técnicas para el diagnóstico de IT. Estos datos se mantienen cuando estudiamos la globalidad de la muestra y en los individuos con $>500$ linfocitos $\mathrm{CD}_{4}$, no así en los pacientes con mayor deterioro inmunológico. Con el uso conjunto del
PPD y del antígeno A-60 en los pacientes más inmunodeprimidos ( $<200$ linfocitos $\mathrm{CD}_{4}$ ) encontramos que un $19,2 \%$ presentan positividad en ambas técnicas.

En los 24 meses de seguimiento, 7 pacientes desarrollaron una TB pulmonar, de ellos 4 presentaban una serología positiva frente al antígeno A-60. Asimismo, 4 eran anérgicos, sospechando que esta enfermedad pudo ser debida a una infección reciente y no a una reactivación.

Finalmente, nos llama la atención la limitada rentabilidad de la realización de un Multitext®, para conocer el estado de anergia en los pacientes con un PPD (-), y así racionalizar la decisión de ofertar QP. Sólo el 11,11\% de los pacientes con PPD (-) resultaban positivos para esta técnica, y todos ellos presentaban negatividad en la medición de anticuerpos frente al A-60, por lo que podría resultar más barato y más racional la determinación de anticuerpos frente al A-60, que la realización de esta prueba cara y en ocasiones difícil de interpretación.

Como conclusión y ante estos resultados, podríamos afirmar que en pacientes anérgicos, un modo de racionalizar la QP frente a $M$. tuberculosis es la realización de una determinación de la IgG frente al antígeno A-60 de M. bovis. Su positividad indica, al igual que un PPD (+), al menos un contacto previo con $M$. tuberculosis que hay que valorar a la hora de instaurar una QP. El seguimiento de estos pacientes en el tiempo puede aportarnos más datos, si bien como ya se ha señalado, de los 7 pacientes que han desarrollado TB pulmonar en el seguimiento de 24 meses, 4 de ellos presentaban una serología positiva frente al A-60. Además, de éstos, 4 eran anérgicos. No obstante cabe esperar, que con la instauración de los nuevos tratamientos antiretrovirales (terapia HAART, etc.) pueda cambiar sustancialmente la historia natural de la infección por el VIH, y como tal de la tuberculosis en este colectivo.

\section{AGRADECIMIENTOS}

Este estudio ha sido financiado por una Beca de Investigación en materia de Salud del Gobierno de La Rioja ( $\left.n^{\circ} 494\right)$.

Agradecemos a todo el personal del Servicio de Análisis Clínico del Hospital de La Rioja su colaboración. Asimismo, agradecemos a el Sr. Enrique Ramalle su colaboración para el análisis estadístico.

\section{Bibliografía}

1. Theuer CP, Hopwell PC, Elias D, Schecter GF, Rutherford GW, Chaisson RE. Human immunodeficiency virus infection in tuberculosis patients. J Infect Dis 1990; 162:8-12.

2. Rey R, Ausina V,m Casal M, Cayla J, de March P, Moreno S, et al. Situación actual de la tuberculosis en España. Una perspectiva saniataria en precario respecto a los países desarrollados. Med Clin (Barc) 1995; 105:703-707.

3. Pitchenik AE. Tuberculosis control and the AIDS epidemic in developing countries. Ann Intern Med 1990; 113:89-91.

4. Centers for Disease Control. Tuberculosis an human immunodeficiency virus infection. Recommendations of the Advisory Committee for the Elimination of Tuberculosis (ACET). MMWR 1989; 38:236-238 y 243250.

5. Grupo de Trabajo sobre Tuberculosis. Consenso Nacional para el Control de la Tuberculosis en España. Med Clin (Barc) 1992; 98:24-31.

6. Selwyn PA, Sckell BM, Alcabes P, Friedland GH, Klein RS, Schoenbaum EE. High risk of active tuberculosis in HIV-infected drug users with cutaneous anergy. JAMA 1992; 268:504-509.

7. Graham NM, Nelson K, Solomon L, Bonds M, Rizzo RT, Scavotto J, et al. Prevalence of tuberculin positivity and skin test anergy in HIV-1seropositive and seronegative intravenous drug users. JAMA 1992; 267:369-373.

8. Portu JJ, Pérez JR, Arévalo JM, Aldamiz-Etxebarria M, Agud JM, Ayensa C. Prueba de la tuberculina en infección por VIH. Enf Infecc Microbiol Clin 1996; 14:90-95.

9. Moreno S, Baraia-Etxaburu J, Bouza E, Parras F, Pérez-Tascón M, Miralles P, Vicente T, Alberdi JC, Cosin J, Lopez Gay D. Risk for developing tuberculosis among anergic patients infected with HIV. Ann Intern Med 1993; 119: 194-198.

10. Moreno S. Infección por el virus de la inmunodeficiencia humana y 
tuberculosis: ¿quién debe recibir profilaxis?. Rev Clin Esp 1994; 194: 73-74.

11. Division of Tuberculosis elimination (CDC). Purified Protein Derivate (PPD) Tuberculin Anergy and HIV infection: Guidelines for anergy testing and management of anergic persons at risk of tuberculosis. MMWR 1991; 40/RR-5:27-33.

12. Sánchez Fuentes D, Barragán JM, Garcinuño MA, Martín-Casado M, Martín-Marcos M. Análisis de la decisión de efectuar quimioprofilaxis con isoniacida en pacientes usuarios de drogas por vía por parenteral e infectados por el VIH. Rev Clin Esp 1994; 194:81-86.

13. Centers for Disease Control. Revised classification system for HIV infection and expanded surveillance of definition for AIDS among adolescents and adults. MMWR 1992; 41 (RR-17):1-19.

14. Webster CT, Gordin FM, Matts JP, Korvick JA, Miller C, Muth K, et al. Two stage tuberculin skin testing in individuals with human immunodeficiency virus infection. Am J Respir Crit Care Med 1995; 151:805-808.

15. Navarro V, Guix J, Bernacer B, Nieto A, Borras R, Ferrer C, García de Lomas J, Juan G, Roig P. Tuberculosis e infección por el virus de la inmunodeficiencia humana. Estudio prospectivo de 215 pacientes. Rev Clin Esp 1993; 192:315-320.

16. Markowitz N, Hansen NI, Wilcosky TC, Hopewell PC, Glassroth J, Kvale PA, et al. Tuberculin and anergy testing in HIV-seropositivy and HIV seronegative persons. Ann Intern Med 1993; 119:185-193.

17. Martínez Vazquez JM, Cabarcos Ortiz de Barrón A, Barrio Gómez E. Tuberculosis e infección por VIH: de la clínica a la prevención. An Med Interna (Madrid) 1997; 14:363-368.

18. Ladrón de Guevara MC, González A, Ortega A, Saz JV. Diagnóstico serológico de la tuberculosis pulmonar mediante ELISA y el antígeno A60. Enf Infecc Microbiol Clin 1992; 10:17-19.

19. Ladrón de Guevara MC, Beltrán M, Gutierrez A, Saz JV. Influencia de la quimioterapia en la respuesta de anticuerpos frente al antígeno A60. Enf Infecc Microbiol Clin 1992; 10:26-28.

20. Gómez-Mampaso E, Palenque E, Sánchez M, et al. Detección de anticuerpos IgG frente al anígeno 60 para el diagnóstico de la tuberculosis humana: resultados de un estudio multicéntrico. Rev Esp Microbiol Clin 1991; 6:456-463.

21. López JL, Pardo FJ, García del Busto A, Cascales P, Fandos E, Galiano JV. Serología de la tuberculosis: detección de anticuerpos IgG e IgM. Determinación del valor umbral de IgG. Rev Esp Microbiol Clin 1990; 5: 618-620.

22. Maes R, Homasson JP, Kubin M, Bayer M. Development of an enzyme immunoassay for the serodiagnosis of tuberculosis and mycobacteriosis. Med Microbiol Immunol 1989; 178:323-335.

23. Casal M, Linares MJ. Diagnóstico serológico de la tuberculosis. estudio de la primera prueba comercializada existente (ANDAELISA-TuberculosisR). Rev Esp Microbiol Clin 1988; 491-492.

24. Querol JM, Oltra C, Granda D, Alonso MC, Climent JL, Labrador T, Minguez J. Utilidad de la determinación de IgG e IgM frente al antígeno 60 para el diagnóstico de la tuberculosis torácica. An Med Interna (Madrid) 1993; 10:271-274.

25. Máttar S, Broquetas J, Sauleda J, Carceller A, Gea J, Aran X, Torres JM. Detección de anticuerpos IgG con el método ELISA en pacientes tuberculosos utilizando el antígeno 60. Rev Esp Microbiol Clin 1989; 2:97-102.

26. Saz JV, Ladrón de Guevara MC. IgG frente al antígeno A60 y pruebas de la tuberculina en individuos sanos y enfermos tuberculosos. Enf Infecc Microbiol Clin 1992; 10:477-480.

27. Maes R. Clinical usefulness of serological measurements obtained by antigen 60 in mycobacterial infection: Development of a new concept. Klin Kochenschr 1991; 69:696-709. 